\title{
Perfil clínico e epidemiológico dos pacientes oncológicos na unidade de atendimento imediato
}

\author{
Clinical and epidemiological profile of the oncology patients in the immediate care unit
}

Perfil clínico y epidemiológico de pacientes oncológicos en la unidad de atención inmediata

João Victor Moura Garcia ${ }^{1 *}$, Maria Theresa Santiago Ribeiro Rodrigues², Rafaela Alves Lima ${ }^{2}$, Thalyta Mariany Rêgo Lopes ${ }^{3}$, Maicon de Araujo Nogueira ${ }^{3}$, Everson Vando Melo Matos ${ }^{2}$, Aline Maria Pereira Cruz Ramos ${ }^{4}$.

\section{RESUMO}

Objetivo: Traçar as características clínicas e epidemiológico dos pacientes oncológicos atendidos na Unidade de Atendimento Imediato (UAI) de um hospital referência em oncologia no Estado do Pará. Método: Estudo descritivo, retrospectivo, transversal com abordagem quantitativa, realizado com 600 pacientes oncológicos atendidos na UAI de um hospital de referência, no período de outubro a dezembro de 2017. Foram realizadas análises descritivas e aplicado o teste qui-quadrado de Pearson. A pesquisa foi submetida e aprovada pelo Comitê de Ética em Pesquisa da UNIFAMAZ sob o parecer de número: 2.037.237. Resultados: Quanto ao perfil epidemiológico predominaram pacientes do gênero feminino (56\%), na faixa etária de $36-55$ anos (42,3\%). Em relação a classificação de risco recebida o maior quantitativo foi na cor verde (pouco urgente) (76\%), a queixa principal mais recorrente foi a dor $(49,8 \%)$. No que diz respeito ao diagnóstico, identificou-se prevalência de câncer de colo do útero $(39,8)$, mama $(17,5 \%)$ e estômago $(4,8 \%)$ no gênero feminino. No gênero masculino, observou-se a predominância de câncer de próstata (20,5\%), laringe (9,5\%) e pele (8,3\%). Conclusão: O estudo permitiu caracterizar o perfil dos pacientes atendidos na unidade referida, servindo como instrumento para melhoria da assistência do serviço e como subsídio para futuros estudos desta natureza.

Palavras-chave: Neoplasias, Emergências, Acolhimento, Perfil de Saúde, Institutos do Câncer, Sinais e Sintomas.

\begin{abstract}
Objective: To describe the clinical and epidemiological characteristics of oncology patients attended at the Immediate Care Unit (UAI) of a reference hospital in oncology in the State of Pará. Method: A descriptive, retrospective, cross-sectional study with a quantitative approach performed with 600 cancer patients attended at UAI of a reference hospital, from October to December 2017. Descriptive analyzes were performed and Pearson's chi-square test was applied. The research was submitted and approved by the Research Ethics Committee of the UNIFAMAZ under the opinion of number: 2.037.237. Results: The epidemiological profile was predominantly female $(56 \%)$, in the age range of $36-55$ years $(42,3 \%)$. Regarding the classification of risk received, the largest quantitative was in the green color (low urgency) (76\%), the main recurrent complaint was pain (49.8\%). Regarding the diagnosis, the prevalence of cervical (39.8), breast (17.5\%) and stomach (4.8\%) cancer were predominant in the female gender. In the male gender was observed the predominance of prostate cancer $(20.5 \%)$, larynx $(9.5 \%)$ and skin (8.3\%) was observed. Conclusion: The study allowed characterizing the profile of the patients treated in the referred unit, serving as an instrument to improve the service assistance and as a subsidy for future studies of this nature.
\end{abstract}

Key words: Neoplasms, Emergencies, User Embracement, Health Profile, Cancer Care Facilities, Signs and Symptoms.

\section{RESUMEN}

Objetivo: Trazar las características clínicas y epidemiológicas de los pacientes oncológicos atendidos en la Unidad de Tratamiento Inmediato (UAI) de un hospital referencia en oncología en el Estado de Pará. Método: Estudio descriptivo, retrospectivo, transversal con abordaje cuantitativo, realizado con 600 pacientes oncológicos atendidos en la UAI de un hospital de referencia, en el período de octubre a diciembre de 2017. Se realizaron análisis descriptivos y aplicados la prueba qui-cuadrado de Pearson. La investigación fue sometida y aprobada por el Comité de Ética en Investigación de la UNIFAMAZ bajo el parecer de número: 2.037.23. Resultados: En

${ }^{1}$ Universidade da Amazônia (UNAMA), Belém-PA. *E-mail: joaovgarcia98@gmail.com

${ }^{2}$ Centro Universitário Metropolitano da Amazônia (UNIFAMAZ), Belém- PA.

3Universidade do Estado do Pará (UEPA), Belém- PA.

${ }^{4}$ Universidade Federal do Pará (UFPA) e Universidade da Amazônia (UNAMA), Belém- PA. 
cuanto al perfil epidemiológico predominaron pacientes del género femenino (56\%), en el grupo de edad de 36 55 años (42,3\%). En relación a la clasificación de riesgo recibida el mayor cuantitativo fue en color verde (poca urgencia) $(76 \%)$, la queja principal más recurrente fue el dolor $(49,8 \%)$. En lo que se refiere al diagnóstico, se identificó la prevalencia de cáncer de cuello de útero $(39,8)$, mama $(17,5 \%)$ y estómago $(4,8 \%)$ en el género femenino. En el género masculino, se observó la predominancia de cáncer de próstata $(20,5 \%)$, laringe $(9,5 \%)$ y piel $(8,3 \%)$. Conclusión: El estudio permitió caracterizar el perfil de los pacientes atendidos en la unidad referida, sirviendo como instrumento para mejorar la asistencia del servicio y como subsidio para futuros estudios de esta naturaleza.

Palabras clave: Neoplasias, Urgencias Médicas, Acogimiento, Perfil de Salud, Instituciones Oncológicas, Signos y Síntomas.

\section{INTRODUÇÃO}

A qualidade em serviços e sistemas de saúde tornou-se tema central e frequente nas discussões em todo 0 mundo. A qualidade e segurança formam um binômio que impulsiona as melhorias em estruturas, resultados e processos (SANTOS ML, et al., 2017).

Atualmente, o câncer é a segunda maior causa de morte no Brasil e no mundo, sendo responsável por 9,6 milhões de mortes no ano de 2018 (BRASIL, 2012a, OPAS/OMS, 2018) Tal fato está relacionado ao envelhecimento da população adoção de hábitos não saudáveis, infecções sexualmente transmissíveis, acesso ineficaz a serviços de saúde para detecção precoce e tratamento associado ao aumento das doenças crônicodegenerativas são fatores que predispõem uma maior probabilidade de desenvolvimento de câncer. Em virtude da abrangência desta patologia é necessária a utilização de instrumentos que visem a melhoria da qualidade da assistência com objetivo de maximizar os benefícios aos pacientes (MIRANDA B et al., 2016).

No Brasil, o Instituto Nacional de Câncer José Alencar Gomes da Silva (INCA) (INCA, 2017) estimou para o biênio 2018-2019, a ocorrência de 600 mil novos casos de câncer por cada ano, excluindo os casos de câncer de pele não melanoma. Espera-se uma taxa de incidência para homens de 217.27/100 mil habitantes e para mulheres de 191.78/100 mil habitantes, apresentando dados semelhantes à de países desenvolvidos. Esses dados são baseados no perfil de um país, no qual prevalece a incidência de canceres de pulmão, próstata, mama feminina, cólon e reto, contudo tal perfil epidemiológico muda-se na região norte e nordeste, as quais além de apresentarem os cânceres já citados entre os principais, tem incidência significativa os cânceres de estômago e de colo do útero. Destaca-se que a região norte apresenta taxas equivalentes de cânceres de mama feminina e de colo do útero.

Estudos demonstram que a maioria dos pacientes com câncer já necessitou de assistência imediata pelo menos uma vez durante o desenvolvimento e o tratamento da doença (GABRIEL J, 2012). Sabe-se que novas estratégias têm sido abordadas para melhoria da qualidade de vida desses pacientes, no entanto, tais terapêuticas também trazem consigo efeitos colaterais, associado a isto há casos de doença avançada, falta do controle da sintomatologia ou complicações que deprimem o estado geral dos pacientes, exigindo atuação de profissionais capacitados para realizar atendimento de boa qualidade seja no âmbito hospitalar, ambulatorial, serviços de urgência e emergência ou na atenção básica (BOAVENTURA AP et al., 2015; MIRANDA ML et al., 2016).

Diante disso, diversas são as conquistas das políticas públicas de saúde consolidas pelo Sistema Único de Saúde (SUS), sendo a estruturação dos serviços de urgência e emergência um exemplo disto. Visando a melhoria da qualidade do serviço, o Ministério da Saúde (MS) instituiu em 2004, a Política Nacional de Humanização (HumanizaSUS), e em 2009 por meio da diretriz do HumanizaSUS sobre o Acolhimento e Classificação de Risco nos Serviços de Urgência (BRASIL, 2004; BRASIL, 2009).

O acolhimento pode ser realizado por qualquer profissional da equipe multiprofissional de saúde, entretanto cabe ao enfermeiro através da Consulta de Enfermagem realizar a Classificação de Risco, por meio de um protocolo com cores que representam a gravidade e urgência no atendimento (vermelho: emergência; amarelo: urgência; verde: pouco urgente; e azul: não urgente), estruturando assim a ordem de atendimento (FEITOSA MM et al., 2017).

A superlotação dos serviços de emergência, definido como o excesso de demanda frente aos recursos disponíveis, compromete o atendimento prestado e configura um problema de saúde pública em diversos países (YARMOHAMMADIAN MH et al., 2017). Dessa forma, percebe-se, que a estratégia de Acolhimento com Classificação de Risco contribui a qualidade assistencial, por se nortear pela escuta qualificada, construção de vínculo, garantia de acesso com responsabilidade de resolutividade dos problemas, e por meio da priorização dos atendimentos mais graves, favorecendo a definição de estratégias quanto aos recursos materiais, humanos, redução de superlotações e melhoria assistencial (HERMIDA PMV et al., 2018).

Diante disso, o presente estudo tem como objetivo traçar as características clínicas e epidemiológico dos pacientes oncológicos atendidos na Unidade de Atendimento Imediato (UAI) de um hospital referência em oncologia no Estado do Pará. 


\section{MÉTODO}

Trata-se de um estudo descritivo, quantitativo, retrospectivo, transversal, realizado em maio de 2018 na UAI de um Centro de Atenção à Alta Complexidade em Oncologia (CACON), localizado no município de Belém, Estado do Pará, sendo este o único CACON do estado.

A casuística foi composta das fichas de 600 pacientes, de ambos os gêneros, atendidos no serviço de acolhimento com classificação de risco da UAl em outubro a dezembro de 2017. Dessa forma, foram considerados como critérios de inclusão pacientes com: idade entre 18 - 95 anos; de ambos os gêneros; diagnosticado com câncer; atendidos no período de outubro a dezembro de 2017.

Foram excluídos os pacientes com idade inferior a 18 anos e superior a 95; aqueles que não receberam classificação de risco; aqueles com preenchimento inadequado das fichas acerca do atendimento; aqueles pacientes que buscaram atendimento para consultas de seguimento, realização de exames, hemotransfusão e pulsoterapia.

Este estudo avaliou dados secundários obtidos das fichas clínicas da própria instituição do período referente ao último trimestre de 2017 (outubro, novembro e dezembro). Para tanto, foi utilizado o Termo de Compromisso para Utilização de Dados (TCUD) e dispensado o Termo de Consentimento Livre e Esclarecido (TCLE). Os dados foram coletados pelos pesquisadores por meio de uma ficha de obtenção de dados, que possibilitou a coleta informações com vista a atender aos objetivos do estudo.

As variáveis estudadas foram: gênero, idade, tipo de câncer, queixa principal e cor de classificação de risco no atendimento. Para o cálculo amostral, obteve-se a média histórica do número de pacientes atendidos na UAI do CACON, levando-se em consideração amostras de população finita e procedimentos de amostragem sem reposição, obteve-se um n de 600, o que garante 95\% de confiança e nível de significância de $p \leq 0,05$.

Após a coleta, os dados foram processados pelo software Microsoft Excel 2010 Windows $\AA$, e realizou-se análise por meio de estatística descritiva, sendo as variáveis descritas em frequência absoluta e relativa e apresentados por meio de tabelas e gráficos. Associado a análise descritiva foi utilizado programa estatístico Winpepi, e realizado o teste estatístico Qui-quadrado de Pearson (ABRAMSON JH, 2011)

A pesquisa foi submetida ao Comitê de Ética em Pesquisa (CEP) do Centro Universitário Metropolitano da Amazônia - UNIFAMAZ, no referido município, associado ao Comitê Nacional de Ética em Pesquisa (CONEP) e aprovado com CAAE: 67177717.50000.5701, sob parecer de número: 2.037.237. A construção e desenvolvimento dessa pesquisa respeitaram os pressupostos que regulamentam pesquisa envolvendo seres humanos contidos na resolução ํㅜ 466/2012 e 580/2018 do Conselho Nacional de Saúde (CNS) (BRASIL, 2012b).

\section{RESULTADOS}

Durante o período analisado, foram atendidos 708 pacientes na UAI, deste total, 600 atendiam aos critérios de inclusão e foram analisados. Através da análise dos dados revelou-se predominância do gênero feminino $(56 \%)$ em detrimento do masculino (44\%). Observou-se a média de idade de 57,3 anos $( \pm 15,65)$ e mediana de 60,5 anos (Tabela 1).

Tabela 1 - Características dos pacientes com câncer atendidos na UAI. Belém-PA, 2019.

\begin{tabular}{lcccccc}
\hline \multirow{2}{*}{ Faixa Etária } & \multicolumn{2}{c}{ Total } & \multicolumn{2}{c}{ Masculino } & \multicolumn{2}{c}{ Feminino } \\
\cline { 2 - 7 } & $\mathbf{N}$ & $\%$ & $\mathbf{N}$ & $\%$ & $\mathbf{N}$ & $\%$ \\
\hline $18-35$ anos & 60 & 10,0 & 28 & 10,6 & 32 & 9,5 \\
$36-55$ anos & 210 & 35 & 68 & 25,8 & 142 & 42,3 \\
$56-75$ anos & 249 & 41,5 & 117 & 44,3 & 132 & 39,3 \\
$76-95$ anos & 81 & 13,5 & 51 & 19,3 & 30 & 8,9 \\
Total & $\mathbf{6 0 0}$ & $\mathbf{1 0 0}$ & $\mathbf{2 6 4}$ & $\mathbf{1 0 0}$ & $\mathbf{3 3 6}$ & $\mathbf{1 0 0}$ \\
\hline
\end{tabular}

Fonte: Dados da pesquisa, 2019.

Em relação a classificação de risco, no serviço avaliado é utilizada a classificação de risco do MS que baseia sua estratificação de atendimento com base em quatro cores com seus respectivos significados: vermelho (atendimento emergente), amarelo (atendimento urgente), verde (atendimento pouco urgente ou semi-urgente) e azul (atendimento não urgente) (BRASIL, 2009).

Observou-se que houve predominância de 76,3\% (458) da classificação da cor verde, representado por 208 homens e 250 mulheres. A classificação na cor azul totalizou 18\% (108) da amostra, sendo 40 homens e 68 mulheres. Seguida da classificação da cor amarela 4,8\% dos pacientes (29), com distribuição de gênero relativamente equiparados e por último a classificação vermelha com $0,8 \%$ dos pacientes (5), sendo 2 homens e 3 mulheres (Tabela 2). 
Tabela 2 - Perfil de classificação de risco dos pacientes com câncer atendidos na UAI. Belém-PA, 2019.

\begin{tabular}{|c|c|c|c|c|c|c|c|}
\hline \multirow{2}{*}{ Cor da classificação de risco } & \multicolumn{2}{|c|}{ Total } & \multicolumn{2}{|c|}{ Masculino } & \multicolumn{2}{|c|}{ Feminino } & \multirow{2}{*}{ p-valor } \\
\hline & $\mathbf{N}$ & $\%$ & $\mathbf{N}$ & $\%$ & $\mathbf{N}$ & $\%$ & \\
\hline Vermelho & 5 & 0,8 & 2 & 0,3 & 3 & 0,5 & $0,857^{a}$ \\
\hline Amarelo & 29 & 4,8 & 14 & 2,3 & 15 & 2,5 & $0,639^{a}$ \\
\hline Verde & 458 & 76,3 & 208 & 34,7 & 250 & 41,7 & $0,438^{a}$ \\
\hline Azul & 108 & 18 & 40 & 6,7 & 68 & 11,3 & $0,126^{a}$ \\
\hline Total & 600 & 100 & 264 & 44 & 336 & 56 & - \\
\hline
\end{tabular}

Fonte: Dados da pesquisa, 2019.

a Teste Qui-Quadrado

Para este estudo foi considerado apenas a queixa principal de cada paciente (Tabela 3), entretanto em sua maioria os pacientes apresentavam mais de um sinal e sintoma. Excluíram-se os atendimentos que procuram o serviço para reavaliação médica, realização de exame laboratorial, hemotransfusão e pulsoterapia, totalizando 108 pacientes que não atendiam aos critérios de inclusão do estudo.

Tabela 3 - Principais queixas dos pacientes atendidos na UAI. Belém-PA, 2019.

\begin{tabular}{|c|c|c|c|c|c|}
\hline Queixa principal & $\mathbf{N}$ (Total) & $\%$ (Total) & $\begin{array}{c}\text { Masculino } \\
\mathrm{N}=264\end{array}$ & $\begin{array}{c}\text { Feminino } \\
\mathrm{N}=336\end{array}$ & p-valor \\
\hline Dor & 299 & 49,8 & 138 & 161 & $0,263^{a}$ \\
\hline Astenia & 59 & 9,8 & 28 & 31 & $0,563^{a}$ \\
\hline Reações Adversa de Antibioticoterapia & 54 & 9,0 & 22 & 32 & $0,622^{a}$ \\
\hline Hemorragia & 29 & 4,8 & 10 & 19 & $0,294^{a}$ \\
\hline Dispneia & 21 & 3,5 & 05 & 16 & $0,059^{a}$ \\
\hline Outros & 138 & 23 & 61 & 77 & - \\
\hline Total & 600 & 100,0 & 264 & 336 & - \\
\hline
\end{tabular}

Fonte: Dados da pesquisa, 2019.

a Teste Qui-Quadrado

Quando avaliados os diagnósticos dos pacientes, optou-se por ordená-los em ordem decrescente do número total de atendidos, sem realizar separação segundo gênero, obtendo-se uma caracterização geral dos atendidos (Tabela 4). Contudo, ressalta-se que a incidência dos cânceres diverge entre os gêneros, sendo no feminino os de maiores incidências os canceres de colo de útero, mama e de estômago, enquanto que nos homens prevalece os canceres de próstata, laringe e outras neoplasias de pele. Desta maneira obtém-se um quadro de incidência diferente para cada gênero.

Tabela 4 - Perfil topográfico de câncer identificados nos pacientes do gênero masculino e feminino atendidos na UAl. Belém-PA, 2019.

\begin{tabular}{lcccccc}
\hline \multirow{2}{*}{ Topografia do Câncer } & \multicolumn{2}{c}{ Masculino } & \multicolumn{2}{c}{ Feminino } & Total & \multirow{2}{*}{ p-valor } \\
\cline { 2 - 5 } & $\mathbf{( N = 2 6 4 )}$ & $\mathbf{( \% )}$ & $\mathbf{( N = 3 3 6 )}$ & $\mathbf{( \% )}$ & $\mathbf{( N = 6 0 0 )}$ & \\
\hline Colo uterino & 0 & 0 & 134 & 39,8 & 134 & - \\
Mama & 8 & 3,0 & 59 & 17,5 & 67 & $7,9 \mathrm{e}-12^{\mathbf{a}}$ \\
Próstata & 54 & 20,5 & 0 & 0 & 54 & - \\
Estômago & 20 & 7,5 & 16 & 4,8 & 36 & $0,407^{\mathbf{a}}$ \\
Laringe & 25 & 9,5 & 3 & 0,9 & 28 & $8,2 \mathrm{e}-6^{\mathbf{a}}$ \\
Outras neoplasias de pele & 22 & 8,3 & 4 & 1,2 & 26 & $1,5 \mathrm{e}-4^{\mathbf{a}}$ \\
Reto & 7 & 2,7 & 15 & 4,5 & 22 & $0,094^{\mathrm{a}}$ \\
Hipofaringe & 12 & 4,5 & 1 & 0,3 & 13 & $1,4 \mathrm{e}-3^{\mathbf{a}}$ \\
Boca & 5 & 1,9 & 7 & 2,1 & 12 & $0,602^{\mathbf{a}}$ \\
Brônquios e pulmões & 5 & 1,9 & 7 & 2,1 & 12 & $0,602^{\mathbf{a}}$ \\
Ossos e cartilagens articulares & 10 & 3,8 & 2 & 0,6 & 12 & $0,015^{\mathbf{a}}$ \\
dos membros & 7 & 2,7 & 3 & 0,9 & 10 & $0,178^{\mathbf{a}}$ \\
Cólon & 2 & 0,8 & 8 & 2,4 & 10 & $0,062^{\mathbf{a}}$ \\
Ânus & 1 & 0,4 & 9 & 2,7 & 10 & $0,012^{\mathbf{a}}$ \\
Cavidade nasal e ouvido médio & 86 & 32,5 & 68 & 20,2 & 154 & - \\
Outras topografias & $\mathbf{2 6 4}$ & $\mathbf{1 0 0}$ & $\mathbf{3 3 6}$ & $\mathbf{1 0 0}$ & $\mathbf{6 0 0}$ & - \\
Total & & & & &
\end{tabular}

Fonte: Dados da pesquisa, 2019.

a Teste Qui-Quadrado 


\section{DISCUSSÃO}

Após a análise e interpretação dos dados coletados, foi possível identificar a faixa etária dos pacientes que utilizam o serviço avaliado, a classificação de risco, queixa principal que levou o paciente a procura do atendimento e a topografia do câncer, dessa forma, é possível definir as características clínicas e epidemiológicas dos indivíduos atendidos.

Neste estudo, houve predominância do gênero feminino convergindo com os resultados obtidos em outro estudo, no qual também foi observado que o seguimento mais atingido é o de mulheres correspondendo a 55,9\% dos pacientes atendimentos nas urgências e emergências, acompanhando o perfil epidemiológico estimado pelo INCA para o presente período (GONÇALVES MM et al., 2018).

A explicação reside no maior quantitativo da população feminina brasileira, além da predominância de mulheres na faixa etária mais elevada. Essa característica representa aquilo que é chamado de processo de feminilização do envelhecimento, comportamento explicado pelo fato de a mortalidade masculina ser superior à feminina, assim como pela maior demanda e maior uso dos serviços de saúde pelas mulheres (SANTOS ML et al., 2017).

A maior demanda dos atendimentos nesse período foi entre a faixa etária 36 a 55 anos e 56 a 75 anos entre mulheres e homens, respectivamente, com média de 57,3 anos, resultado este semelhante ao encontrado em estudo internacional, no qual a idade variou entre 19 a 87 anos, apresentando média de 57,9 anos (SADIK M et al., 2014).

No que tange a classificação de risco, a maioria dos enfermeiros ao utilizar a classificação de risco, classificam $67 \%$ dos pacientes na cor verde, o que significa pouco urgente (OLIVEIRA GN et al., 2011). Da mesma forma, $74,9 \%$ dos atendimentos de um hospital em Minas Gerais, foi em decorrência de afecções que levaram a classificação verde (pouco urgente), e somente $0,1 \%$ dos atendimentos foram classificados com cor vermelha, que representa os casos emergentes, aproximando-se do que foi identificado neste estudo (1\% de classificação da cor vermelha) (MADEIRA DB et al., 2010).

Ressalta-se que neste estudo, foram identificados $94,3 \%$ dos pacientes como de menor complexidade e prioridade (cores verde e azul). Dados similares aos encontrados em estudos que utilizam protocolo institucional que classificam o paciente quanto a cinco prioridades de atendimento (vermelho, laranja, amarelo, verde e azul), como o do Hospital Escola Federal (São Paulo, Brasil) no qual 89,7\% foram classificados como baixo risco e do Serviço de Emergência do Hospital São Paulo, no qual 88,1\% dos pacientes foram classificados como baixo risco (BECKER JB et al., 2015; OLIVEIRA GN et al., 2016)

A dor foi a queixa mais relatada entre os pacientes do serviço nesta pesquisa. Resultados semelhante foram encontrados em um estudo realizado em um hospital público no interior de São Paulo, o qual concluiu que 83,13\% (143 pacientes oncológicos) apresentavam dor como queixa principal, que os levaram ao serviço de emergência (BOAVENTURA AP et al., 2015).

Em estudo internacional realizado em um hospital universitário no sul da Turquia, foram avaliados 408 pacientes no setor de urgências oncológicas cujos apresentavam como sintomas: a falta de ar/dispneia (23,2\%), dor (17,8\%), febre (14,2\%), náusea e vómito (14,4\%) (SADIK M et al., 2014). Em consonância, estudo realizado em um hospital referência no tratamento oncológico no Brasil, demonstrou que o controle da dor é utilizado por $87,1 \%$ dos pacientes com feridas malignas, caracterizando a dor como sintoma presentes em vários estágios de progressão do câncer (RAMOS AMPC et al., 2018).

Devido ao câncer ser uma doença multifatorial, sua sintomatologia é inespecífica e pode ser confundida com outros agravos da saúde, aumentando assim a trajetória entre sintomatologia, diagnóstico e tratamento. Entretanto, Neste estudo foram avaliados somente dados de pacientes diagnosticados com câncer, desta maneira a sintomatologia apresentada por estes indivíduos é semelhante à encontrada na literatura, como: dor, náuseas, fadiga, disfagia, entre outros, sendo estes relacionados com a progressão da doença ou como efeito adverso da terapia antineoplásica, e informados como fator determinante na busca por cuidado (OLIVEIRA JM et al., 2018).

Observou-se a predominância dos cânceres de próstata (20,5\%), laringe (9,5\%, p-valor: 8,2e-6), outras neoplasias de pele (8,3\%, p-valor: 1,5e-4), estômago (7,5\%) e hipofaringe $(4,5 \%$, p-valor: $1,4 \mathrm{e}-3)$ nos pacientes do gênero masculino. Nas pacientes do gênero feminino predominou os canceres de colo uterino (39,8\%), mama (17,5\%, p-valor: 7,9e-12), estômago (4,8\%), reto (4,5\%) e cavidade nasal (2,7\%, p-valor: 0,012). Em contrapartida, estudo internacional apontou que os pacientes oncológicos com intercorrências eram portadores em sua maioria de câncer de pulmão (32,5\%), sistema gastrointestinal $(25,4 \%)$ e câncer de mama (9,3\%) (SADIK $\mathrm{M}$ et al., 2014).

Ratificando os dados de pesquisas internacionais, a Organização Pan Americana da Saúde/Organização Mundial da Saúde (OPAS/OMS) afirma que os seis tipos de canceres mais incidentes na população mundial são os de pulmão, mama, colorretal, próstata, câncer de pele não melanoma e estômago, sendo os de pulmão, colorretal e estômago os com maiores taxas de mortalidade (OPAS/OMS, 2018) Entretanto, os tipos de cânceres mais incidentes neste estudo podem estar relacionado às particularidades da região na qual está situado 0 CACON, sendo estás descrita em outros estudos (INCA, 2017). 
Mesmo apresentando alta prevalência entre os atendidos, alguns tipos de câncer não demonstraram boa significância estatística tais como o câncer de estômago, reto, boca, brônquios e pulmões, entre outros. Tal fato é explicado por esses tipos de câncer serem de grande incidência e atingir em sua maioria a população masculina e feminina com quantitativos proporcionais (INCA, 2017).

Em uma pesquisa realizada em 119 serviços hospitalares do Rio de Janeiro, verificou-se que em todas as regiões, havia superlotação nas emergências. Foi identificado que a principal causa de superlotação foi o atendimento a pacientes com problemas passiveis de solução nos ambulatórios, o que condiz com os dados demonstrados neste estudo (O'DWYER G et al., 2008). Santos et al. destaca que a superlotação dos serviços de urgência é um problema de saúde pública que afeta todos os envolvidos no processo saúde-doença, entretanto é um tema pouco abordado diante de sua importância (SANTOS ETS et al., 2018).

Esse estudo permitiu identificar que a superlotação da UAI pode estar relacionada ao direcionamento equivocado de pacientes que possuem necessidades pouco urgentes e que poderiam ser encaminhados para consultas/procedimentos ambulatoriais. Esse encaminhamento inapropriado ocorre por conta de o CACON ser o hospital que atende esse perfil de paciente com afecções oncológicas no Estado, sendo a oferta deste serviço totalmente atendida por meio do SUS e ser o único CACON no Estado. Vale ressaltar que atendimentos desta natureza também são realizados nas Unidades de Assistência de Alta Complexidade em Oncologia - UNACON e em serviços da rede particular distribuídos pelo estado

O principal tipo de câncer mais prevalente na população masculina e feminina foi o de próstata e o de colo de útero, respectivamente. Entretanto, por se tratar de um tipo de câncer especifico do gênero, não foi possível realizar o teste qui-quadrado para obtenção do $p$-valor, uma vez que há casos somente em uma das populações estudadas.

De acordo com os dados obtidos neste estudo, não houve significância estatística entre os sintomas apresentados e o gênero dos pacientes, demonstrando assim a homogeneidade da sintomatologia do câncer independente do gênero. Observou-se ainda que não houve grau de significância estatística entre a classificação de risco recebida e o gênero.

No âmbito das limitações do estudo, pode ser citado o fato de a instituição não utilizar a classificação de cinco cores geralmente adotadas por outras instituições objetos de estudo. Deve-se considerar também que só foram utilizados dados secundários, e que estes podem ter sido preenchidos de maneira incompleta, causando viés em pesquisas desta natureza. A ausência de sistema informatizado na instituição estudada também configura uma limitação do estudo, uma vez que não é possível identificar o tempo de espera para classificação de risco, tempo de duração da classificação de risco, tempo entre classificação de risco e o primeiro atendimento, entre outros.

\section{CONCLUSÃO}

Os dados permitiram caracterizar o perfil epidemiológico dos pacientes com câncer, atendidos no serviço de acolhimento e classificação de risco da UAl, oferecendo dados com potencial para subsidiar estudos futuros e implantação de medidas gerenciais tendo em vista a melhoria da qualidade e segurança do atendimento no serviço que foi cenário do estudo. Esses resultados poderão subsidiar o planejamento dos cuidados e a atuação da equipe multidisciplinar, permitindo melhorar a qualidade e a segurança no atendimento aos pacientes no hospital do estudo. Vale ressaltar que este se trata de um estudo inédito, pois não há nenhum estudo que descreva o perfil dos atendidos em um CACON da região norte.

\section{REFERÊNCIAS}

1. ABRAMSON JH. WINPEPI updated: computer programs for epidemiologists, and traching potential. Epidemiologic Perspectives \& Innovations. 2011; 8: 1-9.

2. BECKER JB, et al. Triage at the Emergency Department: association between triage levels and patient outcome. Rev Esc Enferm USP. 2015; 49 (5): 783-789.

3. BOAVENTURA AP, et al. Perfil dos pacientes oncológicos atendidos em uma unidade de emergência. Ciencia y Enfermeria. 2015; 21(2): 51-62.

4. BRASIL. Características dos Indicadores. Fichas de Qualificação da RIPSA - 2012 > Mortalidade. Mortalidade proporcional por grupo de causas - C.4. DATASUS - Departamento de Informática do SUS / Ministério da Saúde. Brasília-DF. 2012a.

5. BRASIL. Conselho Nacional de Saúde. Resolução 466/12. Trata de pesquisas em seres humanos e atualiza a resolução 196. Diário Oficial da União. 2012b dez 12.

6. BRASIL. Ministério da Saúde. Secretaria de Atenção à Saúde. Política Nacional de Humanização da atenção e Gestão do SUS. Acolhimento e classificação de risco nos serviços de urgência. Brasília: Ministério da Saúde. 2009.

7. BRASIL. Ministério da Saúde. Secretaria Executiva. Núcleo técnico da Política Nacional de Humanização. Humaniza SUS: Política Nacional de Humanização: a humanização como eixo norteador das práticas de atenção e gestão em todas as instâncias do SUS. Brasília: Ministério da Saúde.

8. FEITOSA MM, et al. Reception with risk rating in ready care unit: an experience report. Rev Enferm UFSM. 2017; 7(1): 136-143.

9. GABRIEL J. Acute oncological emergencies. Nursing Standard. 2012; 27(4): 35-42.

10. GONÇALVES MM, et al. Profile of care measures provided to câncer patients in emergency care unit. Revista de Enfermagem do Centro-Oeste Mineiro. 2018; 8(2595): 1-10. 
11. HERMIDA PMV, et al. User embracement with risk classification in an emergency care unit: an evaluative study. Rev Esc Enferm USP. 2018; 52(03318): 1-7.

12. INSTITUTO NACIONAL DE CÂNCER JOSÉ ALENCAR GOMES DA SILVA - INCA. Coordenação de Prevenção e Vigilância. Estimativa 2018: incidência de câncer no Brasil / Instituto Nacional de Câncer José Alencar Gomes da Silva. Coordenação de Prevenção e Vigilância. - Rio de Janeiro: INCA; 2017.

13. MADEIRA DB, et al. Classificação de risco: perfil do atendimento em um hospital municipal do leste de Minas Gerais. Revista Enfermagem Integrada - Ipatinga: Unileste-MG. 2010; 3 (2): 543-553.

14. MIRANDA B, et al. Cancer patients, emergencies service and provision of palliative care. Rev Assoc Med Bras. 2016; 62(3): 207-211.

15. O'DWYER G, et al. Avaliação dos serviços hospitalares de emergência do estado do Rio de Janeiro. Ciências e Saúde Coletiva. 2008; 13(5): 1637-1648.

16. OLIVEIRA GN, et al. Correlation between classification in risk categories and clinical aspects and outcomes. Rev. LatinoAm Enfermagem. 2016; 24(2842): 1-9.

17. OLIVEIRA GN, et al. Perfil da população atendida em uma unidade de emergência referenciada. Rev Latino-Am Enfermagem. 2011; 19(3): 1-9.

18. OLIVEIRA JM et al. Search for oncological care: perception of patients and Family members. J Nurs UFPE online. 2018; 12(4): 938-946.

19. OPAS/OMS - Organização Pan-Americana de Saúde/Organização Mundial de Saúde. Determinantes Sociais e Riscos para a Saúde, Doenças Crônicas não transmissíveis e Saúde Mental. Folha informativa - Câncer. Brasília, DF, Brasil. 2018.

20. RAMOS AMPC, et al. Characterization of oncological patients with malignant wounds. International Journal of Development Research. 2018; 8(10): 23359-23364.

21. SADIK M, et al. Attributes of cancer patients admitted to the emergency department in one year. World J Emerg Med. 2014; $5(2): 85-89$.

22. SANTOS ETS, et al. Acolhimento com avaliação e classificação de risco: Frente a superlotação dos serviços hospitalares de urgência. Cadernos de graduação. Ciências Biológicas e de Saúde Unit, Aracaju-PI. 2018; 5(1): 187-202.

23. SANTOS ML, et al. Epidemiological profile of patients seen in the pre-anesthetic assessment clinic of a university hospital. Rev Bras Anestesiol. 2017; 67(5): 457-467.

24. YARMOHAMMADIAN MH, et al. Overcrowding in emergency departments: a review of strategies to decrease future challenges. J Res Med Sci. 2017; 16; 22:3. 